\title{
Response to Letter to the Editor by Dr. Schulz-Stubner et al.
}

\section{Slippery Platelet Syndromes in Subdural Hematoma}

\author{
Paul T. Akins
}

Published online: 14 September 2010

(C) Springer Science+Business Media, LLC 2010

\section{Dear Editor:}

Dr. Shulz-Stubner makes an excellent point that desmopressin can quickly reverse platelet dysfunction, through its action to release von Willebrand factor. Since the desmopressin effect is short-acting and neurosurgical re-bleeding carries such adverse consequences, we view this treatment as emergent and temporizing until fresh platelets can be transfused.

The caution to watch for water retention after desmopressin is quite germane in neurocritical care patients. This can lead to hyponatremia with additional adverse effects on brain edema and risk of seizures.
P. T. Akins $(\bowtie)$

Neurocritical Care, Kaiser Permanente, Sacramento, CA, USA

e-mail: akins@surewest.net

\section{P. T. Akins}

Department of Neurosurgery, University of California,

San Francisco, CA, USA 